\title{
Teucrium polium L. Improved Heart Function and Inhibited Myocardial Apoptosis in Isolated Rat Heart Following Ischemia-Reperfusion Injury
}

\author{
Maryam Mahmoudabady ${ }^{1,2}$, Faezeh Sadat Talebian', Narges Amel Zabihi', Seyed \\ Abdolrahim Rezaee ${ }^{3}$, Saeed Niazmand ${ }^{1 *}$
}

\begin{abstract}
1 Department of Physiology, Faculty of Medicine, Mashhad University of Medical Sciences, Mashhad, Iran
${ }^{2}$ Neurogenic Inflammation Research Center, Mashhad University of Medical Sciences, Mashhad, Iran

${ }^{3}$ Immunology Research Center, Mashhad University of Medical Sciences, Mashhad, Iran
\end{abstract}

\section{Key Words}

ischemia-reperfusion injury, apoptosis, Teucrium, heart

\section{Abstract \\ Objectives: Myocardial reperfusion is the only logical cure for ischemic heart disease. However, ischemic-rep- erfusion (I/R) injury is one of the underlying factors facilitating and accelerating the apoptosis in the my- ocardium. This study set to investigate the impact of Teucrium polium (TP) hydro-alcoholic extract on I/R induced apoptosis in the isolated rat heart.}

Methods: Isolated rat hearts were classified into six groups. The control samples were subjected to $80 \mathrm{~min}$ of perfusion with Krebs-Henseleit bicarbonate (KHB) buffer; in control-ischemia group, after primary perfusion $(20 \mathrm{~min}$ ) the hearts were exposed to global ischemia (20 min) and reperfusion (40 min). Pretreated groups were perfused with $500 \mu \mathrm{M}$ of vitamin $\mathrm{C}$ and various TP concentrations $(0.5,1,2 \mathrm{mg} / \mathrm{ml})$ for $20 \mathrm{~min}$, and then the hearts were exposed to ischemia and reperfusion for 20 min and 40 min, respectively. Cardiodynamic parame-

Received: Dec 03, 2017 Reviewed: Aug 02, 2018 Accepted: Aug 14, 2018

$@$ This is an Open-Access article distributed under the terms of the Creative Common Attribution Non-Commercial License (http://creativecommons.org/licenses/by-nc/4.0/) which permits unrestricted noncommercial use, distribution, and reproduction in any medium, provided the original work is properly cited.

( This paper meets the requirements of KS X ISO 9706, ISO 9706-1994 and ANSI/NISO Z39.48-1992 (Permanence of Paper). ters including rate pressure product (RPP), heart rate (HR), the maximum up/down rate of left ventricular pressure $( \pm d p / d t)$, left ventricular developed pressure (LVDP), and coronary artery flow (CF) were achieved from Lab Chart software data. The Bax and BCl-2 gene expressions were measured in heart samples.

Results: Hearts treated with TP extract and vit C represented a meaningful improvement in cardiac contractile function and CF. The overexpression of Bcl-2, downregulation of Bax, and improvement of apoptotic index (Bax/Bcl-2) were observed in pretreated TP extract and vit $\mathrm{C}$ hearts.

Conclusion: The TP extract was found to ameliorate the cardiac function in the reperfused myocardium. Also, it can hinder apoptotic pathways causing cardioprotection.

\section{Introduction}

Cardiovascular events are the major reasons for mortality and morbidity throughout the world. Coronary heart ailment is a critical stimulant of mortality among cardiovascular disorders [1].

Prosperous myocardial reperfusion through primary

*Corresponding Author

Saeed Niazmand. Department of Physiology, Faculty of Medicine, Mashhad, 9177948564, Iran.

Tel: +98-513-800-2223 Fax: +98-513-882-8564

E-mail: niazmands@mums.ac.ir 
percutaneous coronary intervention or thrombolytic treatment is the most beneficial procedure for reduction of the myocardial infarct size and improvement of the clinical result after an acute myocardial infarction. Reperfusion can, however, intensify the myocardial injury by ischemia/ reperfusion (I/R) damage [2]. I/R injury causes a cascade of critical pathogenic cellular incidences like mitochondrial injury, calcium massive influx, and speedy depletion of high stores of energy, excessive reactive oxygen species (ROS) abundance, inflammatory reactions causing apoptotic and necrotic cell death [3].

The oxidative stress by ROS is found crucial in such injury which can lead to myocardial cell apoptosis and subsequently impairs cardiac function. Apoptosis also plays an important role in I/R injury development. Anti-apoptotic agents are able to save the heart from I/R incidence, and also this mechanism is a crucial factor in this injury [4]. Since prevention and management of myocardial I/R injury is a decisive concern in coronary heart disease surgery, the anti-apoptosis medicines from plants show a sensible therapeutic procedure to cure I/R injury.

Teucrium polium L. (Lamiaceae), $20-50 \mathrm{~cm}$ high, is a perennial shrub, spread broadly in dry and rocky parts of hills and deserts of South Western Asia, Europe, Mediterranean countries, and North Africa. Oblong, sessile, and linear leaves are almost $3 \mathrm{~cm}$ long. T. polium locally named kalpooreh is extensively found in Iran, and it has small cluster of pink to white flowers with an aromatic odor originated from its bruised foliage [5]. According to phytochemical experiments, $T$. polium possesses different compounds involving flavonoids, terpenoids, and iridoids [6]. T. polium is used for more than 2000 years because of having tonic, antipyretic, diuretic, antispasmodic, diaphoretic, and cholagogic features $[7,8]$. The plant also has insulinotropic, hypoglycemic, and anti-inflammatory functions [9] as well as antinociceptive and antioxidant properties [10-12]. There is increasing evidence regarding cardiovascular effects of T. polium such as positive inotropic and chronotropic effects [13], reduction of blood pressure [14], and lowering blood lipid [15]. Nevertheless, whether or not agents derived from plants, which have antioxidant function, may decrease myocardial apoptosis by I/R and thus enhance ventricular function has not been directly investigated.

Thus regarding the properties of this plant and our previous experience based on antioxidant effects of this plant on improving cardiodynamic factors, the current study evaluated the influences of T. polium on cardiac functional factors and apoptosis triggered by I/R injury in the isolated hearts of rats.

\section{Material and Methods}

\subsection{Plant material and preparation of the extract}

The aerial part of $T$. polium was collected in autumn from the Khorasan Province, Ferdows, Iran, and detected by herbalists in Ferdwosi University of Mashhad, Iran. (Voucher No. 152-2016-4). Then, it was left to dry at room temperature in a dry and dark room. $300 \mathrm{~g}$ of the plant was soaked in ethanol (50\%) for $48 \mathrm{~h}$. Then, it was evaporated and filtrated at $40^{\circ} \mathrm{C}$ for $72 \mathrm{~h}$. The dried extract was dissolved in distilled water to make various concentrations $0.5,1$, and $2 \mathrm{mg} / \mathrm{ml}[14,16]$.

\subsection{Animals and Experimental Design}

60 Wistar male rats weighing 250-280g (from animal house of Mashhad University of Medical Sciences (mums)) were classified into six groups, $\mathrm{n}=10$ for each. The following groups were involved in study: groups of control, control-ischemia (CI), Ext 0.5, Ext 1, Ext 2, and Vit C.

The rats were maintained under standard laboratory conditions: at $22 \pm 4^{\circ} \mathrm{C}$, relative humidity of $60 \pm 15 \%$, and natural light-dark photoperiod. All treatments were conducted based on the Animal Experimentation Ethics Committee of mums (approval no. 930677).

The animals were heparinized (375 units/200g) and anesthetized using intraperitoneal ketamine injection $(60 \mathrm{mg} /$ $\mathrm{kg}$ ). After tracheotomy and opening the chest, the hearts were quickly cut out and cooled down in ice-cold saline until the contractions ceased. Hearts were then put on a Langendorff device and isovolumically perfused at a fixed pressure $(70 \mathrm{mmHg})$ using Krebs-Henseleit bicarbonate buffer (K-H buffer) of this composition (in mmol/L): $\mathrm{NaCl}$, 120; $\mathrm{HNaCO} 3,25 ; \mathrm{KCl}, 4.8$; MgSO4, 1.33; $\mathrm{KH} 2 \mathrm{PO} 4,1.2$; $\mathrm{CaCl} 2$, 1.6; Na2 EDTA, 0.02; and glucose, 10. The perfusate was gassed via $95 \% \mathrm{O} 2$ and $5 \% \mathrm{CO} 2(\mathrm{pH} 7.4)$ and maintained at a fixed temperature $\left(37^{\circ} \mathrm{C}\right)$. Control hearts were perfused uninterruptedly via K-H buffer purely for $80 \mathrm{~min}$. Hearts of CI group were firstly perfused for $20 \mathrm{~min}$, and then the infusion was suspended for $20 \mathrm{~min}$ and reperfusion for $40 \mathrm{~min}$. Hearts in the treated groups were perfused firstly for $20 \mathrm{~min}$ instead of K-H buffer with various T. polium extract concentrations $(0.5,1$, and $2 \mathrm{mg} / \mathrm{ml})$ and Vitamin C (Vit C) $(500 \mu \mathrm{M})$. Afterwards, the infusion was suspended for $20 \mathrm{~min}$ and reperfusion was continued for 40 min with different concentrations of $T$. polium extract and Vit C. Vitamin C was used as a positive control [17] and for comparing its efficacy as an antioxidant with that of $T$. polium.

\subsection{Measurement of Cardiodynamic Parameters}

For measuring pressures of left ventricular, the left atrium was exposed, and a latex balloon attached to pressure transducer was embedded in the left ventricle via the mitral valve. The balloon volume was set to achieve $10 \mathrm{mmHg}$ left ventricular end-diastolic pressure. The myocardial performance indices were left ventricular developed pressure (LVDP), described as peak systolic pressure minus end diastolic pressure, contraction peak rate $(+d p / d t \max$ ),relaxation peak rate(- $d p / d t$ min),heart rate(HR; cardiac spontaneous rhythm was counted per min), and the rate pressure product $(\mathrm{RPP}=\mathrm{LVDP} \times \mathrm{HR})$, gained by a digital data acquisition system (AD instrument, Australia). Coronary flow (CF) was calculated using timed collections of the coronary effluent during all experiment phases. At the end of experiments, the isolated hearts were preserved in RNA later solution at $-70^{\circ} \mathrm{C}$. 


\subsection{RNA Extraction and cDNA Synthesis}

Total RNA was isolated from homogenized rat heart applying reagent of Trizol (Roche Diagnostics GmbH, Mannheim, Germany), based on the manufacturer's instruction. The samples' quality and quantity were indicated spectrophotometrically applying A260 and A260/280 ratio (NanoDrop ND-1000, Thermo Scientific, Delaware, USA) and assessed through visualizing ribosomal bands $28 \mathrm{~S}$ and $18 \mathrm{~S}$ with ethidium bromide after electrophoretic isolation over formaldehyde denaturing $1.2 \%$ agarose gel.

The first-strand cDNA was reverse-synthesized by the RevertAid $^{\mathrm{TM}}$ First-Strand cDNA Synthesis Kit (Fermentas, Germany) along with random hexamer primers according to the instructions of the manufacturer.

\subsection{Quantitative Real-Time PCR (qPCR)}

The primers of quantitative real time PCR Syber Green method were designed for complete cDNA of Bcl-2, Bax, and $\beta$-actin based on sequence data available on NCBI databases utilizing Beacon Designer (PREMIER Biosoft International, Palo Alto, CA; version 7). Primers' specificity were checked by BLAST analysis (NCBI). A quantitative real-time PCR was done by Syber Green Master Mix (Takara Biotechnology, Otsu and Shiga, Japan). The primers' specificity and reliability was approved via performing endpoint PCR and amplicon sequencing through DNA sequencing, Applied Biosystems (SEQLAB, Germany). The designed primers are summarized in Table 1. $\beta$-actin was utilized as housekeeping gene for the normalization of data. Relative two standard curves real time PCR Syber Green method analyzed gene expressions in a Rotor Gene Q 6000 (Qiagen Hilden, Germany). The data were analyzed using the 6-point 10 -fold serial dilution standard curves for genes of interest and reference gene. Standard curves were plotted for target and reference genes, and then Rotor Gene 6000 software (Qiagen, Germany) was used to analyze the data. The relative quantity of Bcl-2 and Bax gene expressions were normalized to the $\beta$-actin mRNA relative quantity and expressed as gene expression index.

\subsection{Statistical Analysis}

SPSS 20 (IBM SPSS Inc., USA) was employed for analysis. Values are expressed as mean \pm standard error mean (SEM). One-way ANOVA was used for statistical assessment followed by the Tukey's test to compare the groups. $P<0.05$ was assumed statistically meaningful.

\section{Results}

Since the changes in all cardiac parameters were almost similar in all the time before ischemia and those after reperfusion, here, only the average changes $2 \mathrm{~min}$ prior to ischemia and the first $10 \mathrm{~min}$ of reperfusion period are mentioned.

\subsection{The Effects of T. Polium on Cardiac Function and Coronary Flow (CF)}

Compared to the CI group, the heart rate in all extract groups and Vit $\mathrm{C}$ decreased by $6 \mathrm{~min}$ after reperfusion $(\mathrm{P}<0.05)$, but then they showed a significant increase $\mathrm{P}<0.05)$; this increasing trend continued by the end of the reperfusion period (Figure 1).

During the first $10 \mathrm{~min}$ after the establishment of reperfusion, LVDP and RPP in all groups of extract and Vit C increased in comparison to the CI group $(\mathrm{P}<0.05$ to $\mathrm{P}<0.001$; Figures $1 \mathrm{~b}$ and $3 \mathrm{a})$.

$+\mathrm{dp} / \mathrm{dt}$ max also showed increased trend during the first 10 min of reperfusion in Ext 0.5, Ext 1, and Vit C groups compared to CI group $(\mathrm{P}<0.05$ to $\mathrm{P}<0.001)$. On the other hand, - dp/dt min in all groups of extract decreased compared to the CI group; however, these reductions had different trends, and maximum changes were observed in Ext 0.5 group $(\mathrm{P}<0.05$ to $\mathrm{P}<0.01$; Figures $2 \mathrm{a}$ and $2 \mathrm{~b})$.

Figure $3 \mathrm{~b}$ indicates the elevated trend of $\mathrm{CF}$ in all groups of the extract and Vit $\mathrm{C}$ compared to the CI group during the first 10 min of reperfusion $(\mathrm{P}<0.05$; Figure $3 \mathrm{~b})$.

Table 1 Designed primers for semi-quantitative real-time polymerase chain reaction.

\begin{tabular}{ll}
\hline Gene & Primers sequences \\
\hline Bcl-2 & Forward: 5'- GTG GTG GAG GAA CTC TTC -3' \\
& Reverse: 5'- GAT CCA GGT GTG CAG ATG -3' \\
& Forward: 5'- AGG GTT TCA TCC AGG ATC -3' \\
Bax & Reverse: 5'- TCC ACA TCA GCA ATC ATC -3' \\
& Forward: 5'- ATT GCT GAC AGG ATG CAG AAA -3' \\
$\boldsymbol{\beta}$-actin & Reverse: 5'- TAG AGC CAC CAA TCC ACA CAG -3' \\
\hline
\end{tabular}




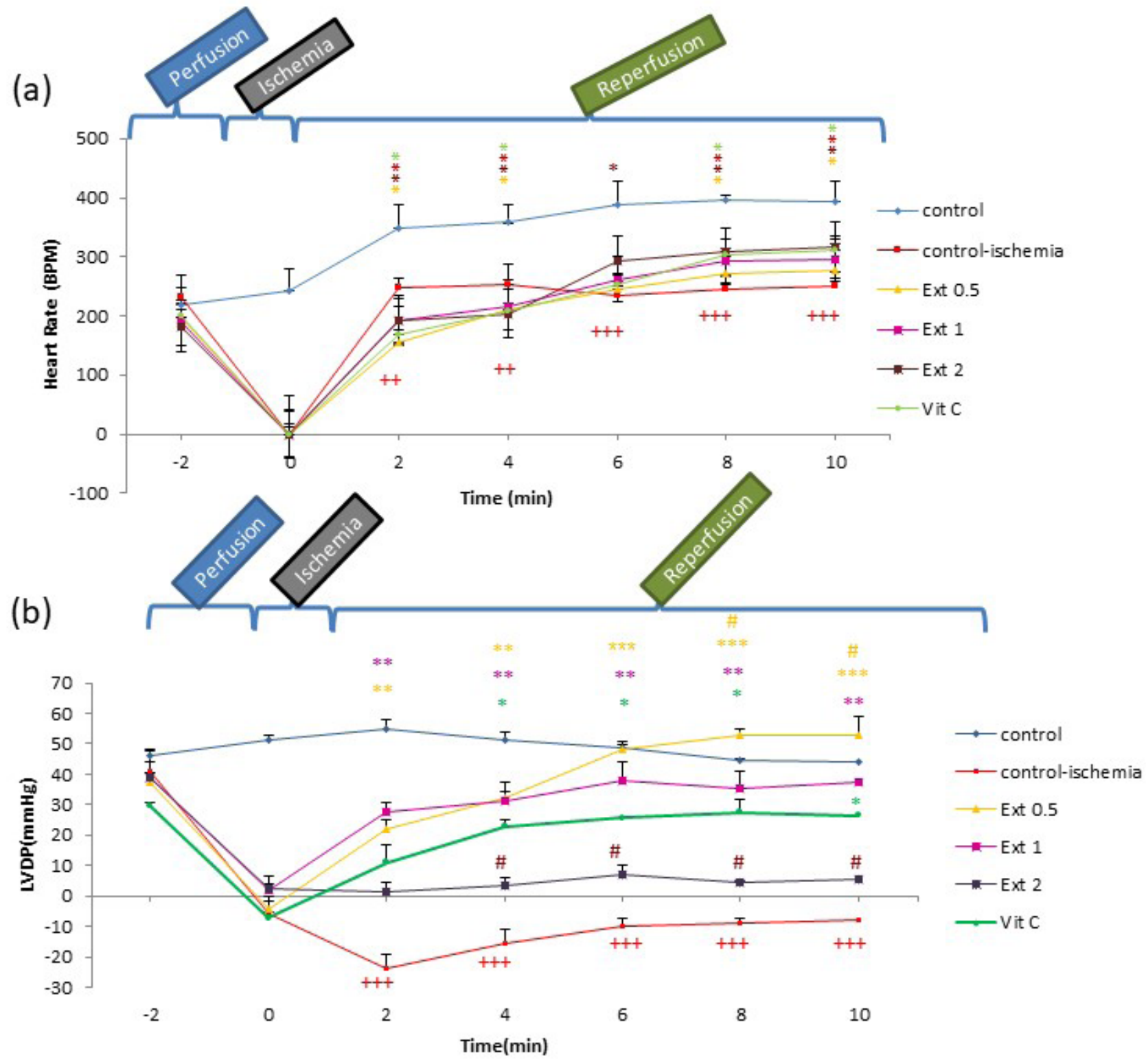

Figure 1 Time course of change in heart rate (HR) and left ventricular developed pressure (LVDP) in control, control ischemia, different concentrations of Teucrium polium extract $(0.5,1$ and $2 \mathrm{mg} / \mathrm{ml}$; Ext 0.5, Ext 1, Ext 2$)$ and Vit C pretreated rat groups during ischemia-reperfusion. The data provided are mean \pm SEM. $+++p<0.001$ compared to control group. ${ }^{*} p$ $<0.05,{ }^{* *} p<0.01,{ }^{* * *} p<0.001$ compared to control ischemia group and $\# p<0.05$ compared to Vit $\mathrm{C}$ group. 

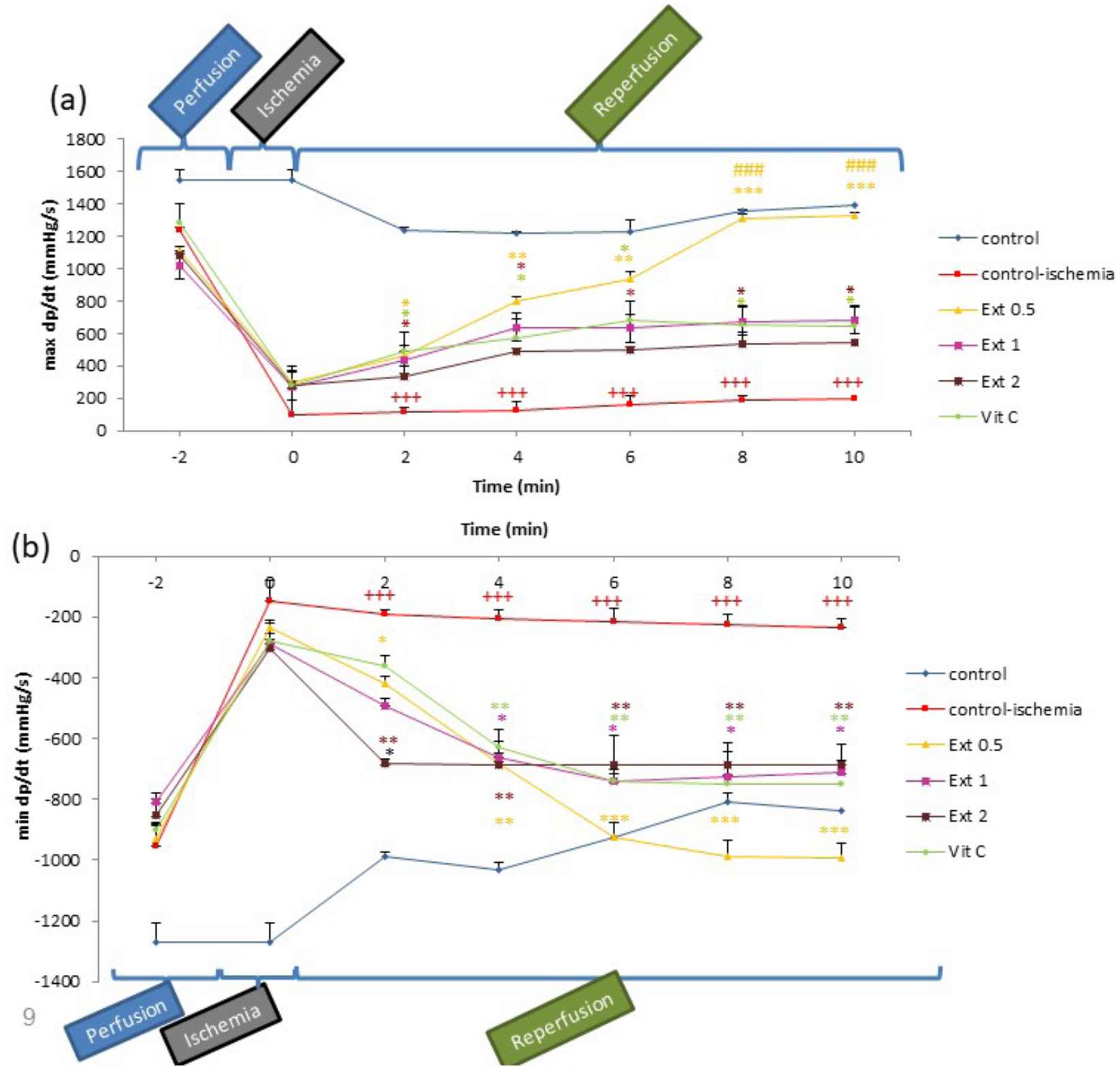

Figure 2 Time course of change in the rate of ventricular pressure development $(+\mathrm{dp} / \mathrm{dt})$ and the rate of ventricular pressure decline (-dp/dt) in control, control ischemia, different concentrations of Teucrium polium extract $(0.5,1 \mathrm{and} 2 \mathrm{mg} /$ $\mathrm{ml}$; Ext 0.5, Ext 1, Ext 2) and Vit C pretreated rat groups during ischemia-reperfusion. The data provided are mean $\pm \operatorname{SEM}$. $+++p<0.001$ compared to control group. ${ }^{*} p<0.05,{ }^{* *} p<0.01,{ }^{* * *} p<0.001$ compared to control ischemia group and \#\#\# $<0.001$ compared to Vit $\mathrm{C}$ group. 


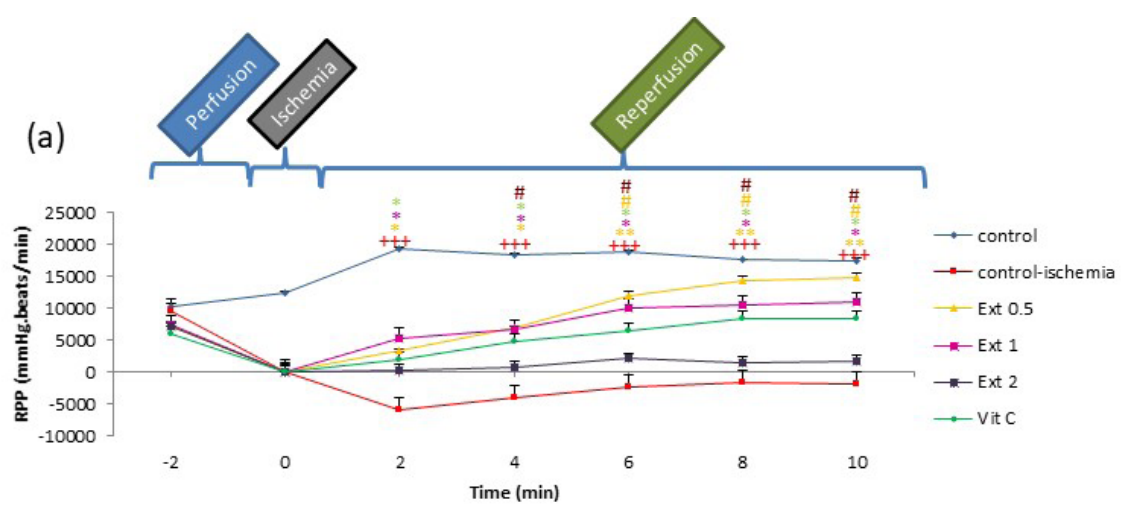

(b)

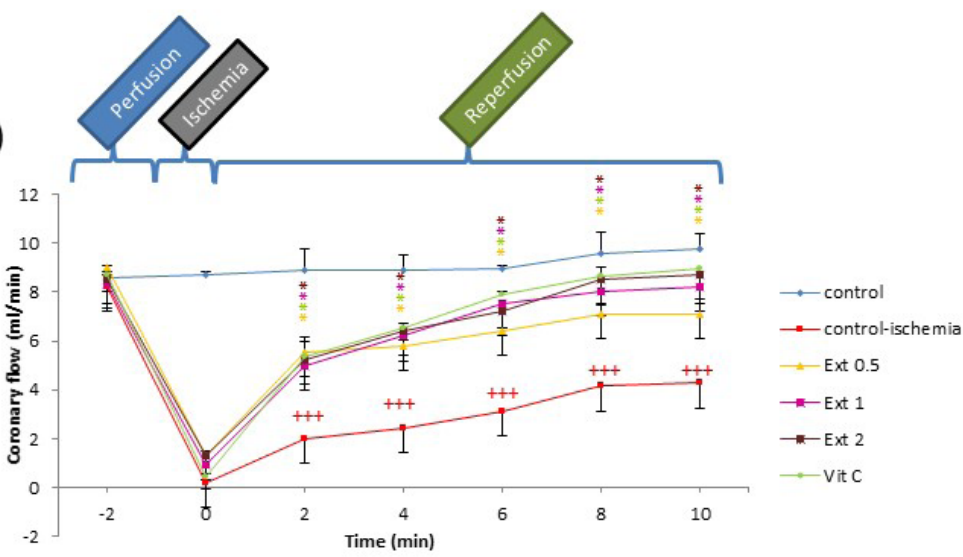

Figure 3 Time course of change in rate pressure product (RPP) and coronary flow (CF) in control, control ischemia, different concentrations of Teucrium polium extract (0.5, 1 and $2 \mathrm{mg} / \mathrm{ml}$; Ext 0.5, Ext 1, Ext 2) and Vit C pretreated rat groups during ischemia-reperfusion. The data provided are mean \pm SEM. $+++p<0.001$ compared to control group. ${ }^{*} p<0.05,{ }^{* *} p$ $<0.01$ compared to control ischemia group and $\# p<0.05$ compared to Vit $C$ group.
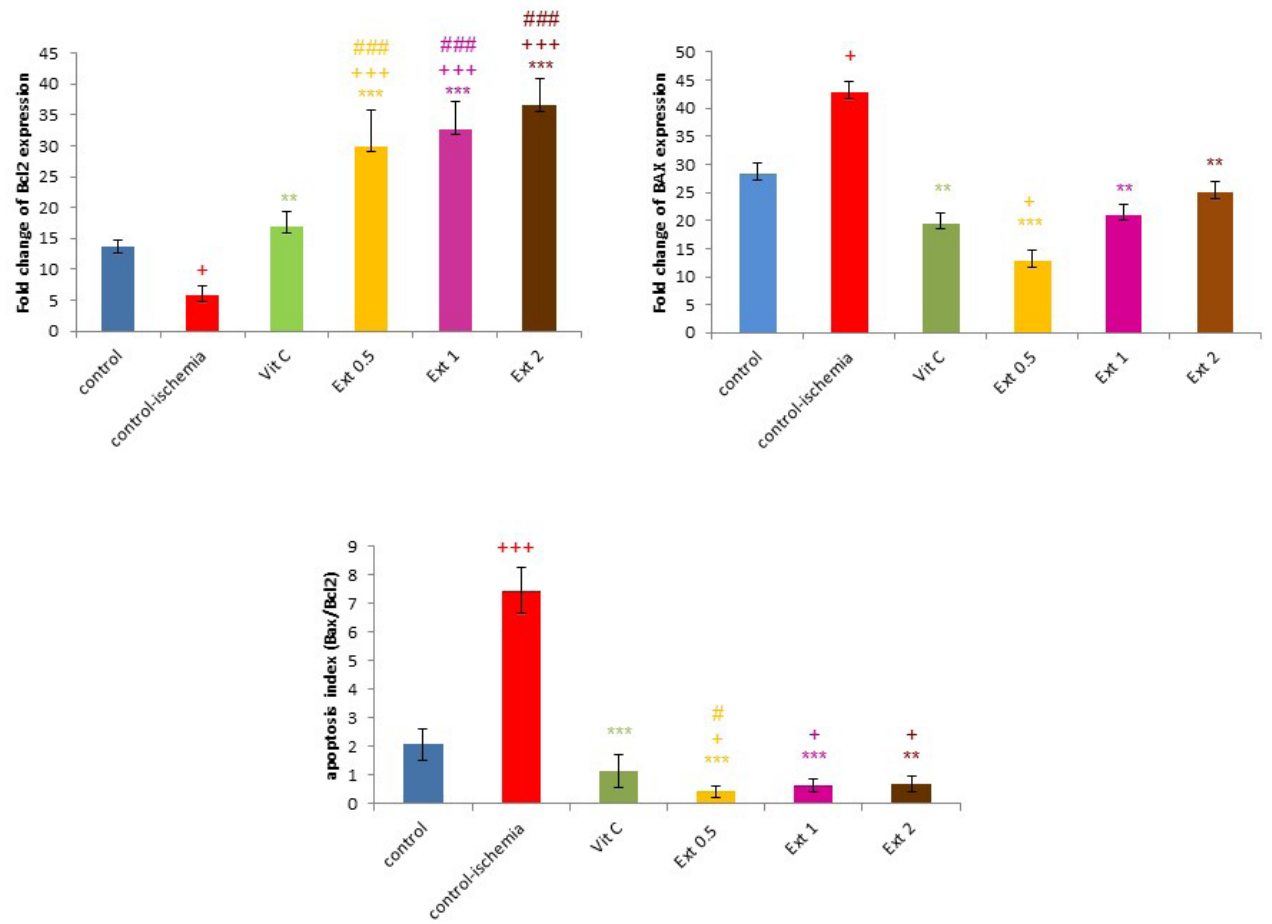

Figure 4 The Fold change of Bcl-2, Bax and Bax/Bcl-2 gene expression in control, control ischemia, different concentrations of Teucrium polium extract $(0.5,1$ and $2 \mathrm{mg} / \mathrm{ml}$; Ext 0.5, Ext 1, Ext 2$)$ and Vit $C$ pretreated rat groups during ischemia-reperfusion. The data provided are mean \pm SEM. $+p<0.05,++p<0.01,+++p<0.001$, compared to control group. ${ }^{*} p<0.05,{ }^{* *} p<0.01,{ }^{* * *} p<0.001$ compared to control ischemia group, \#\# $p<0.01$ and \#\#\# $p<0.001$ compared to Vit $\mathrm{C}$ group. 


\subsection{Bcl-2 and Bax Gene Expressions}

Figure 4a demonstrates that the gene expression of Bcl-2 in CI group declined in comparison to the control $(\mathrm{P}<0.05)$, while this gene expression showed a significant increase in all treated extract groups and Vit C in comparison to CI group $(\mathrm{P}<0.01$ to $\mathrm{P}<0.001)$.

On the other hand, the gene expression of Bax that was elevated in CI group when compared to the control $(\mathrm{P}<0.05)$ decreased in all treated extract groups and Vit $\mathrm{C}$ in comparison to CI group $(\mathrm{P}<0.01$ to $\mathrm{P}<0.001)$ (Figure $4 \mathrm{~b}$ ).

Accordingly, apoptosis index (Bax/Bcl-2 ratio) decreased in $\mathrm{CI}$ group compared with the control $(\mathrm{P}<0.05)$, but a meaningful increment was seen in all treated extract groups and Vit $\mathrm{C}$ in comparison to $\mathrm{CI}$ group $(\mathrm{P}<0.05$ to $\mathrm{P}<0.001$ ) (Figure 4c).

\section{Discussion}

Myocardial reperfusion following ischemia behaves as a double-edged sword, and in spites its benefits to help myocardium for restoring normal function induces a sequence of events, causing cellular injury and organ disorder [18]. Reperfusion damage starts after oxygen restoration and metabolic substrates to metabolically perturbed and energy-deprived tissue [19]. Myocardial oxidative stress and apoptosis stimulated by I/R are involved in the development and progression of myocardial dysfunction in heart failure. These changes are expressed during ischemia and may be modulated through interventions at the beginning of reperfusion $[20,21]$.

Cardiomyocyte apoptosis is a fundamental form of cell necrosis in ischemia and reperfusion impairment resulting in further injury to the cardiac function, $\mathrm{AV}$ and inter-nodal pathway, conduction disturbances owing to SA degeneration, cardiomyopathy, and cardiac remodeling. Thus, such drastic loss of tissue can seriously affect the ventricle geometry and performance $[22,23]$. Increase in oxidative stress and decrease in antioxidant defense in reperfused ischemic hearts causes cardiac impairment because of apoptosis [24].

Stimulating apoptosis mechanisms across reperfusion have not been comprehensively identified, but the release and/or activation of different bioactive materials such as ROS [25] account for the processes inducing apoptosis in reperfusion. Accordingly, in our previous study [26], amelioration of cardiac function was observed after applying T. polium along with improvement of oxidative balance in the same groups.

Mitochondria are crucial contributors to myocardial damage during reperfusion and ischemia [27]. In apoptotic cardiomyocyte loss, there exist two main apoptotic signaling pathways: extrinsic pathway through Fas ligand or TNF- $a$ and intrinsic pathway through mitochondria. The Bcl-2 family pro-apoptotic members e.g. Bax and Bak improve the mitochondrial outer membrane permeability in the intrinsic pathway, releasing protein including cytochrome $\mathrm{c}$ from the intermembrane space to the cyto- plasm and the anti-apoptotic members e.g. Bcl-2 and Bcl$\mathrm{XL}$ that inhibit this process at several steps [28].

In the present study, the gene expression of Bax and Bcl-2 inducer, and inhibitor of apoptosis were assesses to specify the contribution of apoptosis in I/R stimulated damage. To evaluate whether the apoptosis inhibition has any straightforward impact on cardiac performance, hemodynamic performance was controlled and recorded at preset time points across the experiment.

The results showed that in the control ischemia group, the impairment of myocardial I/R stimulated apoptotic cell loss. Escalade of Bax expression and reduction of Bcl-2 gene expression observed in the control ischemia group in comparison to the control approves the impact of apoptosis in myocardial damage after I/R. This observation confirms premier research studies [29,30]. Additionally, in line with rise in apoptotic cell death, damage of post ischemic reperfusion also caused considerable depression in left ventricular dynamics and CF.

Whilst T. polium treatment significantly attenuated Bax and augmented Bcl-2 gene expressions as well as reduced their ratio (apoptosis index) more than the control ischemia group, emphasizing its prominent anti-apoptotic activity. Although formerly the usage of $T$. polium has been reported to inhibit development of cancer in animal tumor models via its apoptosis-inducing and antiproliferative impacts [31-33], in contrast to prior works, notable anti-apoptotic activity of this herb was seen in the current study.

The cardioprotective impact of T. polium is due to the oxidative stress suppression and is correlated with enhance ventricular performance [34]. Nevertheless, no experimentshavebeenreportedontheassociationbetweentheputativeanti-apoptoticinfluencesof T. polium on the functional improvement of ischemia reperfused myocardium, but there are some evidences which indicate the anti-apoptotic impact of this plant in rat retina and liver $[35,36]$.

The present study also showed that $T$. polium pretreatment preserved left ventricular performance as seen in a meaningful elevation in contractility $(+) \mathrm{dP} / \mathrm{dt}$, relaxation (-) dP/dt, and improvement of HR, LVDP, RPP, and CF. T. polium pretreatment may indirectly have restored the flow of blood in the ischemic areas towards normal condition as evaluated by its efficiency in enhancing cardiac function, particularly, adjusting the increment induced by ischemia and reperfusion in LVDP and RPP.

On the other hand, former studies have been shown that T. polium has inotropic and chronotropic effects $[13,16]$, so improvement of cardiodynamic parameters along with CF recovery in extract groups after reperfusion could be attributed to these properties of plant. Hypotensive[14] and vasodilatory effects of $T$. polium were observed previously in our lab [34], thus the augmentation of CF in extract pretreated groups could be due to this vasorelaxation, mainly mediated by inhibition of calcium influx in vascular smooth muscle cells; moreover, vasodilation in lower concentrations was mediated by nitric oxide and prostacyclin. Considering the decent effects of T. polium on LVDP observed in this work, it seems that the reduction of diastolic LVP ameliorates CF.

The data indicated that the lower concentration of extract 
had more influence on cardiodynamics. Also, apoptosis index in this group showed better progression compared to other groups of extract with higher concentrations and even compared with Vit C. This is in accordance with the results of our precedent research that demonstrated the lower concentration of TP had more effectivity on cardiodynamics and oxidative balance [26]. Former studies have shown cytotoxicity of $T$. polium in different types of cell lines [37], and also hepatotoxicity of this plant has been reported by some investigators [38,39]. On the other hand, since our results rely on in-vitro data and regarding the toxicity of T. polium, for better evaluation the efficiency of this plant, much lower doses of its extract are recommended to be considered in further studies.

Furthermore, a crucial restriction of the present study is the absence of protein measurements regarding the measurements of mRNA content.

\section{Conclusion}

The anti-apoptotic effect of T. polium and the ameliorated ventricular functions propose that pretreatment with this herb may have cardioprotective influences after reperfusion and coronary ligation.

\section{Acknowledgment}

This paper is extracted from a M.Sc. thesis. The authors would like to thank Research Affairs of Mashhad University of Medical Sciences for their financial support (grant No. 930677).

\section{Conflict of interest}

The authors declare that there are no conflicts of interst.

\section{References}

1. Sadeghi N, Dianat M, Badavi M, Malekzadeh A. Cardioprotective effect of aqueous extract of Chichorium intybus on ischemia-reperfusion injury in isolated rat heart. Avicenna journal of phytomedicine. 2015;5(6):568.

2. Tian X-h, Liu C-l, Jiang H-L, Zhang Y, Han J-c, Liu J, et al. Cardioprotection provided by Echinatin against ischemia/reperfusion in isolated rat hearts. BMC cardiovascular disorders. 2016;16(1):119.

3. Qu D, Han J, Ren H, Yang W, Zhang X, Zheng Q, et al. Cardioprotective effects of astragalin against myocardial ischemia/reperfusion injury in isolated rat heart. Oxidative medicine and cellular longevity. 2015;2016.

4. Zhu L, Wei T, Gao J, Chang X, He H, Luo F, et al. The car- dioprotective effect of salidroside against myocardial ischemia reperfusion injury in rats by inhibiting apoptosis and inflammation. Apoptosis. 2015;20(11):143343.

5. Bahramikia S, Yazdanparast R. Phytochemistry and medicinal properties of Teucrium polium L. (Lamiaceae). Phytother Res. 2012;26(11):1581-93.

6. Piozzi F, Bruno M, Rosselli S, Maggio A. Advances on the chemistry of furano-diterpenoids from Teucrium genus. Heterocycles 2005;65:1221-34.

7. Said O, Khalil K, Fulder S, Azaizeh H. Ethnopharmacological survey of medicinal herbs in Israel, the Golan Heights and the West Bank region. J Ethnopharmacol. 2002;83(3):251-65.

8. Zargari A. Medicinal Plants. 4th ed. Tehran, Iran: Tehran University Publication; 1996.

9. Esmaeili MA, Yazdanparast R. Hypoglycaemic effect of Teucrium polium: studies with rat pancreatic islets. J Ethnopharmacol. 2004;95(1):27-30.

10. Abdollahi M, Karimpour H, Monsef-Esfehani HR. Antinociceptive effects of Teucrium polium $\mathrm{L}$ total extract and essential oil in mouse writhing test. Pharmacol Res. 2003;48(1):31-5.

11. Ljubuncic P, Dakwar S, Portnaya I, Cogan U, Azaizeh H, Bomzon A. Aqueous extracts of Teucrium polium possess remarkable antioxidant activity in vitro. Evid Based Complement Alternat Med. 2006;3(3):329-38.

12. Zabihi N, Mousavi SM, Mahmoudabady M, Soukhtanloo M, Sohrabi F, Niazmand S. Teucrium polium L. improves blood glucose and lipids and ameliorates oxidative stress in heart and aorta of diabetic rat. . International Journal of Preventive Medicine 2017; In press.

13. Niazmand S, Erfanian Ahmadpoor M, Moosavian M, Derakhshan $\mathrm{M}$. The positive inotropic and chronotropic effects of Teucrium polium L. extract on Guinea pig isolated heart. Pharmacologyonline. 2008;2:588-94.

14. Mahmoudabady M, Shafei MN, Niazmand S, Khodaee E. The Effects of Hydroalchoholic Extract of Teucrium polium L. on Hypertension Induced by Angiotensin II in Rats. Int J Prev Med. 2014;5(10):1255-60.

15. Rasekh HR, Khoshnood-Mansourkhani MJ, Kamalinejad M. Hypolipidemic effects of Teucrium polium in rats. Fitoterapia. 2001;72(8):937-9.

16. Niazmand S, Esparham M, Hassannia T, Derakhshan M. Cardiovascular effects of Teucrium polium L. extract in rabbit. Pharmacogn Mag. 2011;7(27):260-4.

17. Mahmoudabady M, Lashkari M, Niazmand S, Soukhtanloo M. Cardioprotective effects of Achillea wilhelmsii on the isolated rat heart in ischemia-reperfusion. Journal of Traditional and Complementary Medicine 2017; In press.

18. Hausenloy DJ, Yellon DM. Myocardial ischemia-reperfusion injury: a neglected therapeutic target. The Journal of clinical investigation. 2013;123(1):92.

19. Hori M, Nishida K. Oxidative stress and left ventricular remodelling after myocardial infarction. Cardiovascular research. 2008;81(3):457-64.

20. Marczin N, Bundy RE, Hoare GS, Yacoub M. Redox regulation following cardiac ischemia and reperfusion. Coronary artery disease. 2003;14(2):123-33.

21. Wang Q-D, Pernow J, Sjöquist P-O, Rydén L. Phar- 
macological possibilities for protection against myocardial reperfusion injury. Cardiovascular research. 2002;55(1):25-37.

22. Chandrashekhar Y. Role of apoptosis in ventricular remodeling. Current heart failure reports. 2005;2(1):1822.

23. Van Empel VP, Bertrand AT, Hofstra L, Crijns HJ, Doevendans PA, De Windt LJ. Myocyte apoptosis in heart failure. Cardiovascular research. 2005;67(1):21-9.

24. Palojoki E, Saraste A, Eriksson A, Pulkki K, Kallajoki M, Voipio-Pulkki L-M, et al. Cardiomyocyte apoptosis and ventricular remodeling after myocardial infarction in rats. American Journal of Physiology-Heart and Circulatory Physiology. 2001;280(6):H2726-H31.

25. Zhao Z-Q. Oxidative stress-elicited myocardial apoptosis during reperfusion. Current opinion in pharmacology. 2004;4(2):159-65.

26. Mahmoudabady M, Haghshenas M, Niazmand S. Extract from Teucrium polium L. protects rat heart against oxidative stress induced by ischemic-reperfusion injury. Advanced Biomed Research 2017; In press.

27. Lesnefsky EJ, Chen Q, Tandler B, Hoppel CL. Mitochondrial dysfunction and myocardial ischemia-reperfusion: implications for novel therapies. Annual review of pharmacology and toxicology. 2017;57:535-65.

28. Shamas-Din A, Kale J, Leber B, Andrews DW. Mechanisms of action of Bcl-2 family proteins. Cold Spring Harbor perspectives in biology. 2013;5(4):a008714.

29. Galang N, Sasaki H, Maulik N. Apoptotic cell death during ischemia/reperfusion and its attenuation by antioxidant therapy. Toxicology. 2000;148(2):111-8.

30. Maulik N, Yoshida T, Das DK. Oxidative stress developed during the reperfusion of ischemic myocardium induces apoptosis. Free Radical Biology and Medicine. 1998;24(5):869-75.

31. Sghaier MB, Skandrani I, Nasr N, Franca M-GD, Chekir-Ghedira L, Ghedira K. Flavonoids and sesquiterpenes from Tecurium ramosissimum promote antiproliferation of human cancer cells and enhance antioxidant activity: a structure-activity relationship study. environmental toxicology and pharmacology. 2011;32(3):336-48.

32. Tafrihi M, Toosi S, Minaei T, Gohari AR, Niknam V, Arab Najafi SM. Anticancer properties of Teucrium persicum in PC-3 prostate cancer cells. Asia Pac J Cancer Prevent. 2014;15(2):785-91.

33. Rajabalian S. Methanolic extract of Teucrium polium L. potentiates the cytotoxic and apoptotic effects of anticancer drugs of vincristine, vinblastine and doxorubicin against a panel of cancerous cell lines. Exp Oncol. 2008;30(2):133-8.

34. Niazmand S, Fereidouni E, Mahmoudabady M, Hosseini M. Teucrium polium-induced vasorelaxation mediated by endothelium-dependent and endothelium-independent mechanisms in isolated rat thoracic aorta. . Pharmacognosy Research 2017; In press.

35. Aghazadeh S, Yazdanparast R. Inhibition of JNK along with activation of ERK1/2 MAPK pathways improve steatohepatitis among the rats. Clinical nutrition. 2010;29(3):381-5.

36. Dilsiz N, Sahaboglu A, Yıldız MZ, Reichenbach A.
Protective effects of various antioxidants during ischemia-reperfusion in the rat retina. Graefe's Archive for Clinical and Experimental Ophthalmology. 2006;244(5):627-33.

37. Eskandary H, Rajabalian S, Yazdi T, Eskandari M, Fatehi K, Ganjooei NA. Evaluation of Cytotoxic Effect of Teuerium polium on a New Glioblastoma Multiforme Cell Line (REYF-1) Using MTT and Soft Agar Clonogenic Assays. Int J Pharmacol. 2007;3(5):435-7.

38. Mazokopakis E, Lazaridou S, Tzardi M, Mixaki J, Diamantis I, Ganotakis E. Acute cholestatic hepatitis caused by Teucrium polium L. Phytomedicine. 2004;11(1):83-4.

39. Savvidou S, Goulis J, Giavazis I, Patsiaoura K, Hytiroglou P, Arvanitakis C. Herb-induced hepatitis by Teucrium polium L.: Report of two cases and review of the literature. Eur J Gastroenterol Hepatol. 2007;19(6):507-11. 